SUPPORTING INFORMATIONS

\title{
Total synthesis of (+)-oxo-tomaymycin
}

Françoise Benedetti, ${ }^{\dagger}$ Marc-Antoine Perrin,${ }^{\S}$ Sebastien Bosc,${ }^{\ddagger}$ Franck Chouteau, ${ }^{\ddagger}$ Nicolas Champion ${ }^{\ddagger}$ and Antony Bigot $*, \dagger$

antony.bigot@sanofi.com

\section{Table of content}

NMR spectra $\left({ }^{1} \mathrm{H}\right.$ and $\left.{ }^{13} \mathrm{C}\right)$

Preparatives SFC and HPLC data for separation of $(\boldsymbol{E})$ and $(\boldsymbol{Z})-\mathbf{1 1}$

X-Ray crystallography data of (+)-oxo-Tomaymycin 2
S1

S2-S13

S14-S16

S17-20 
NMR

Compound 10 (E/Z): ${ }^{1} \mathrm{H}$ NMR (200 MHz, DMSO d $\left.{ }^{6}\right)$

$\stackrel{\infty}{\stackrel{\infty}{\sim}}$

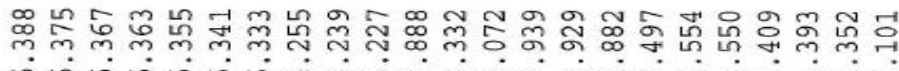

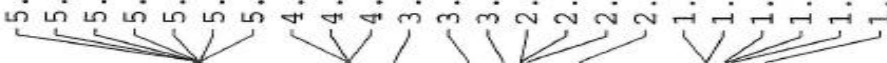

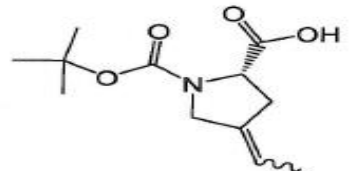

Chemical Formula: $\mathrm{C}_{12} \mathrm{H}_{19} \mathrm{NO}_{4}$
Molecular Weight: 241,29
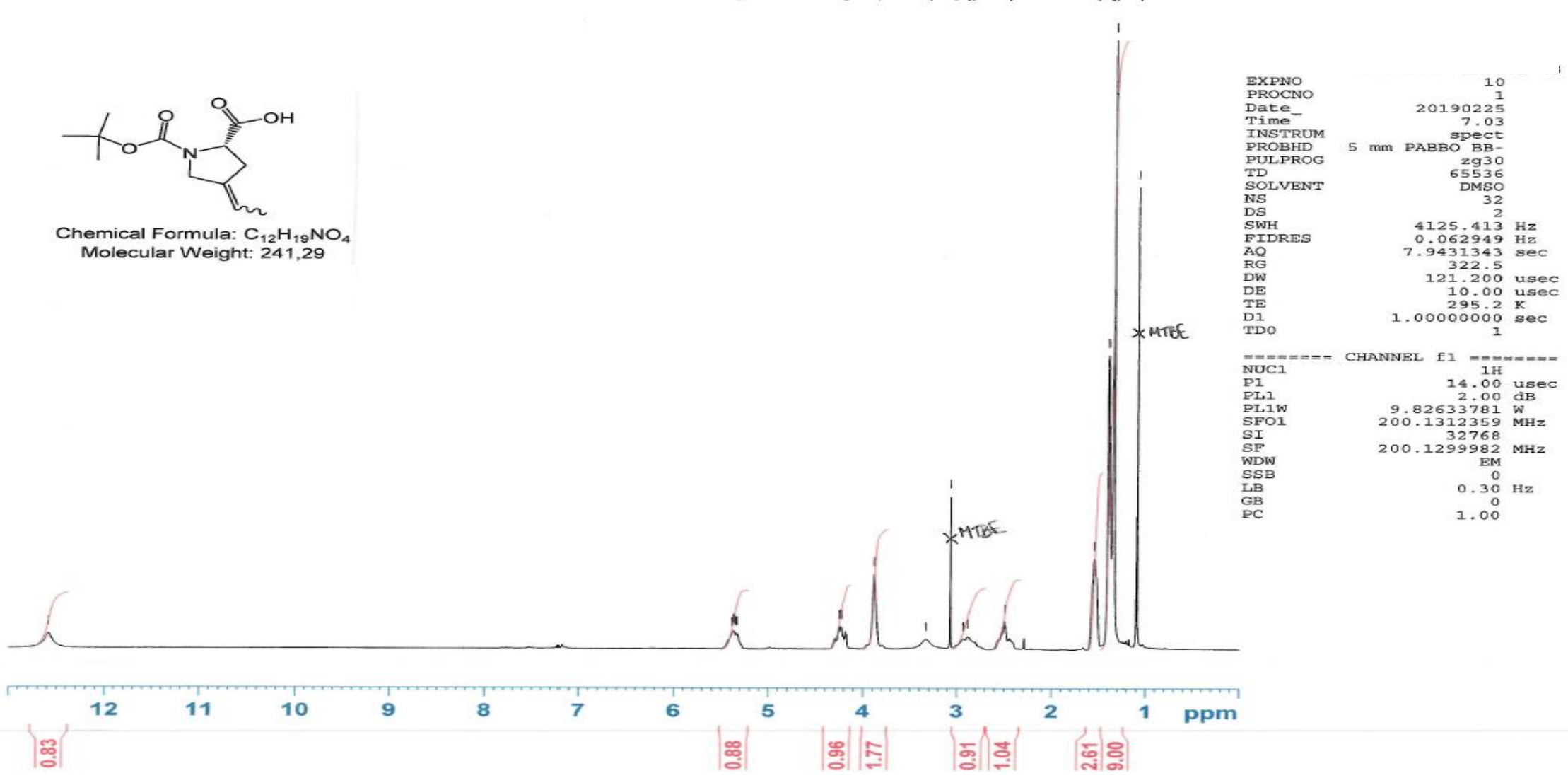


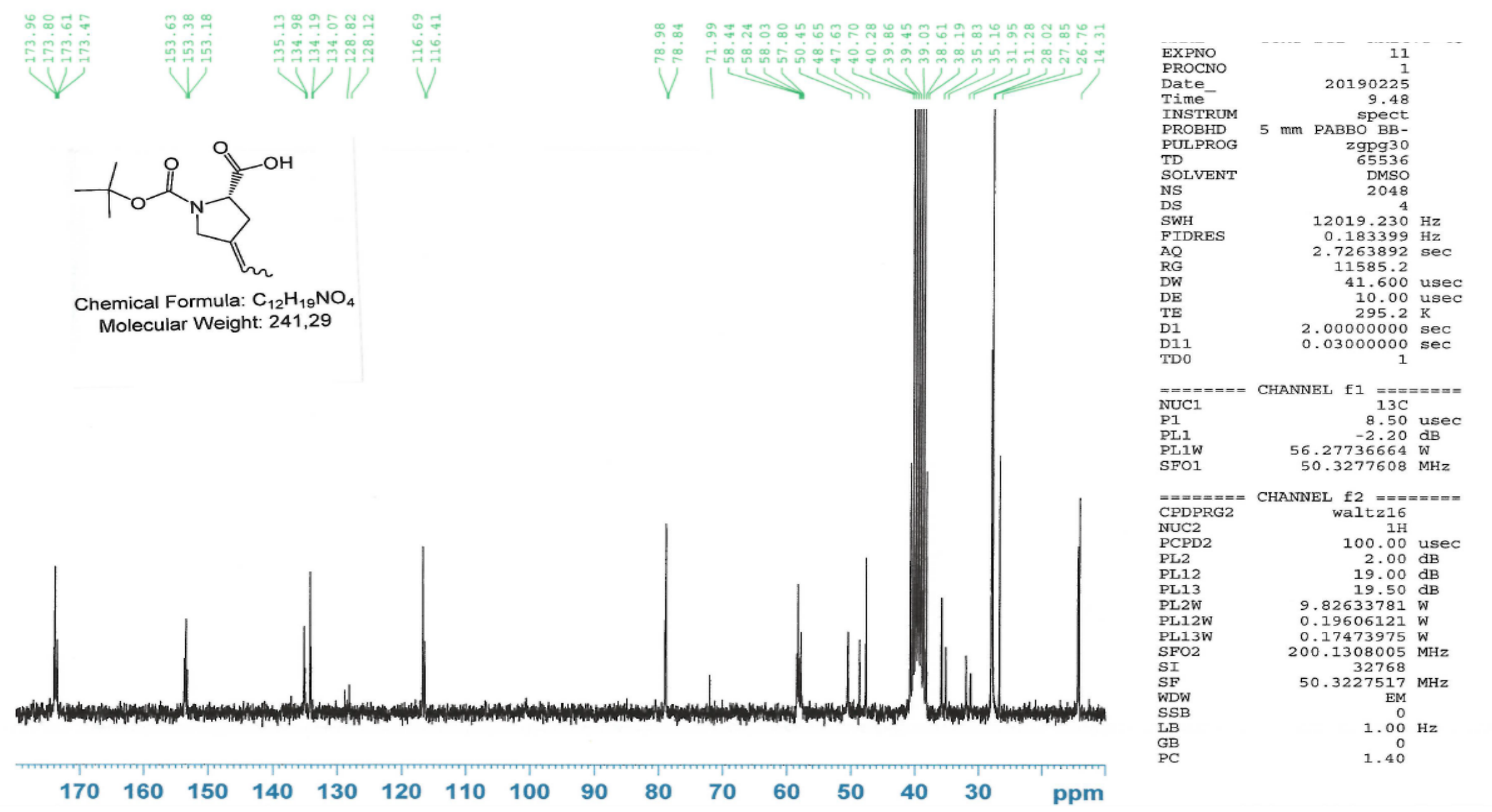




\section{Compound (E)-11: ${ }^{1} \mathrm{H}$ NMR (200 MHz, DMSO d6)}

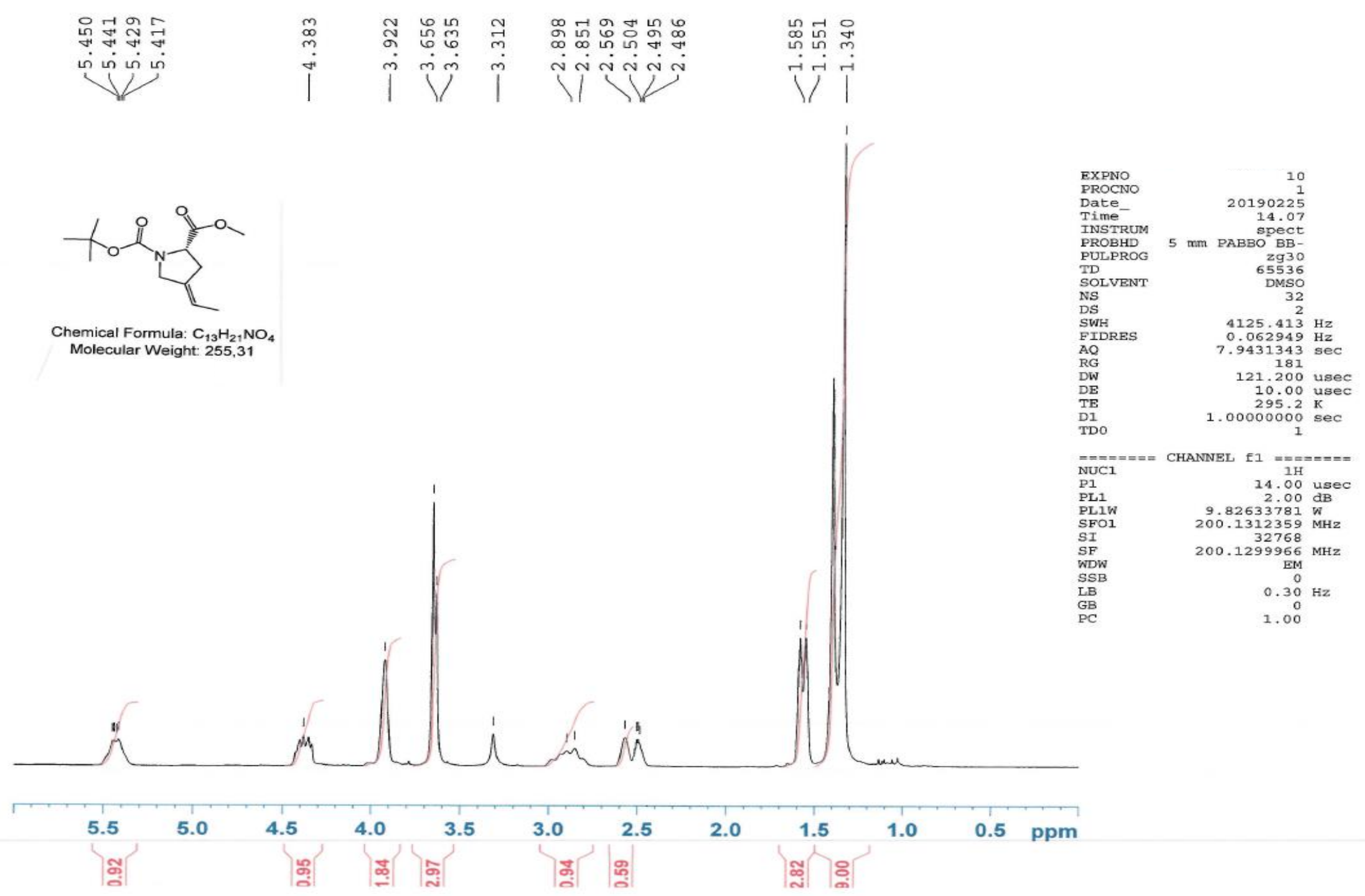


Compound $(\boldsymbol{E})-\mathbf{1 1}:{ }^{13} \mathrm{C}$ NMR (50 MHz, DMSO d6)

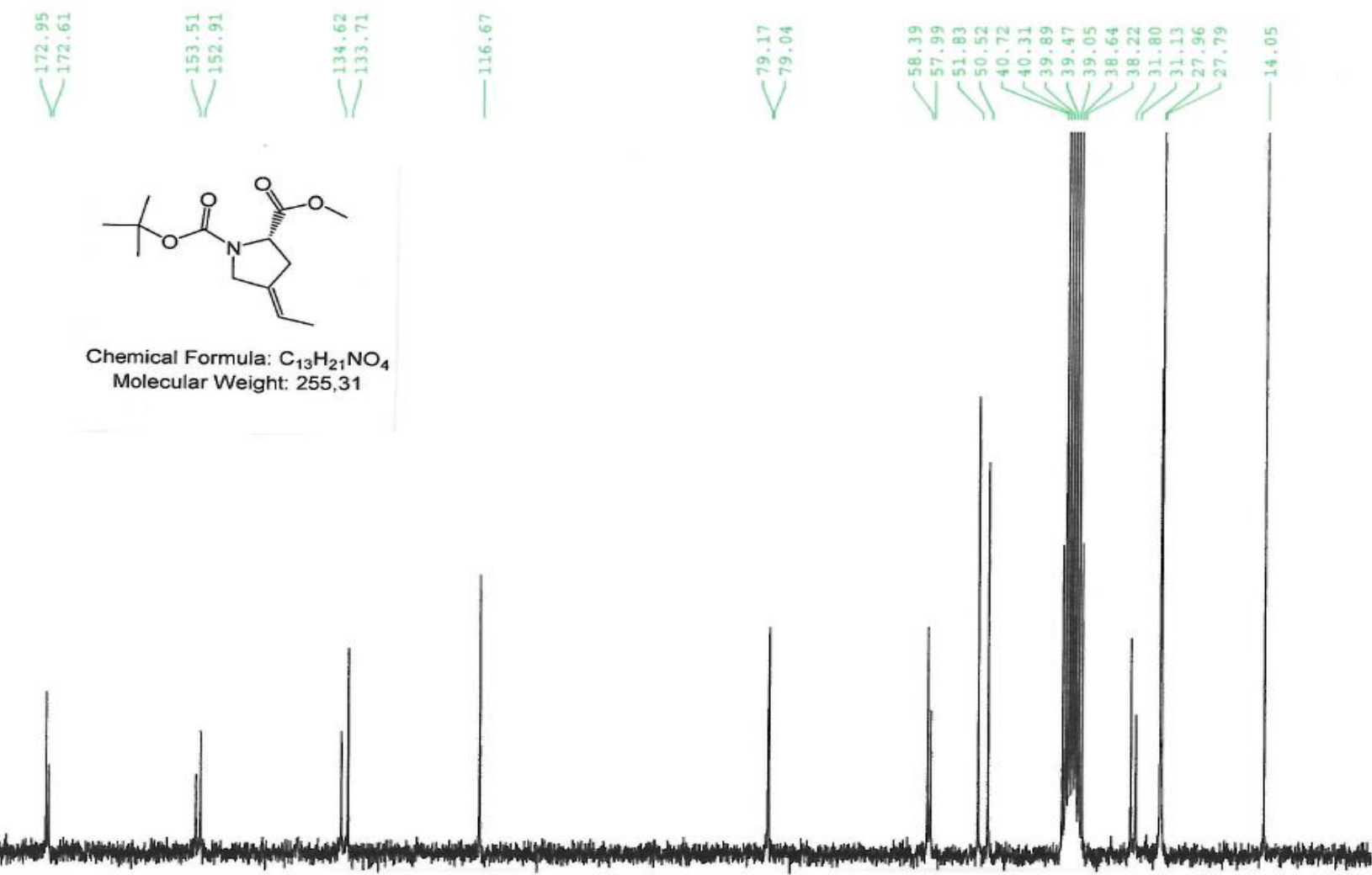

160

140

120

100

80

60

40

20

ppm

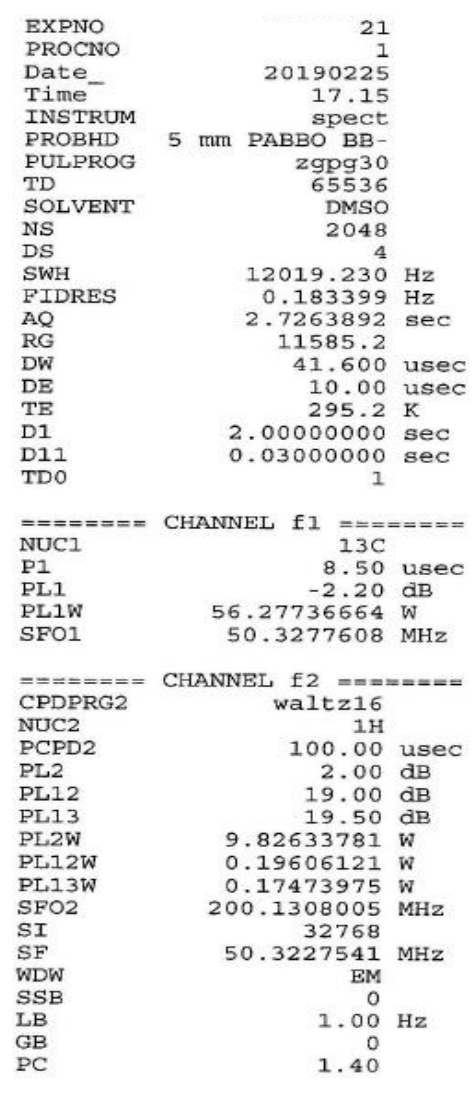




\section{Compound (E)-12: ${ }^{1} \mathrm{H}$ NMR (200 MHz, DMSO d6)}

$\underset{\infty}{\infty}$

ㄱ.

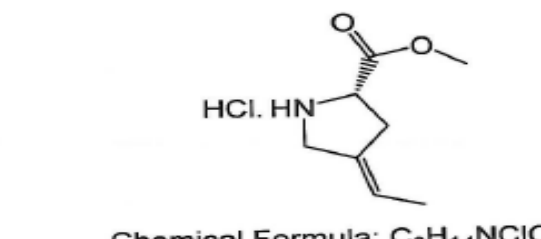
Molecular Weight: 191,70

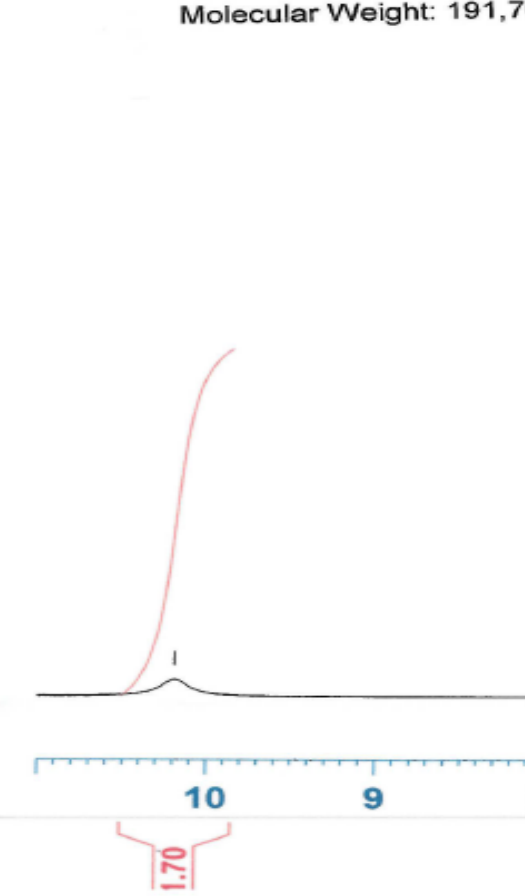

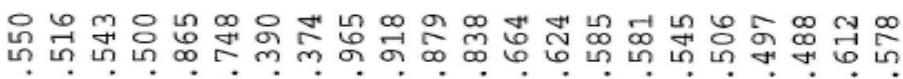
以

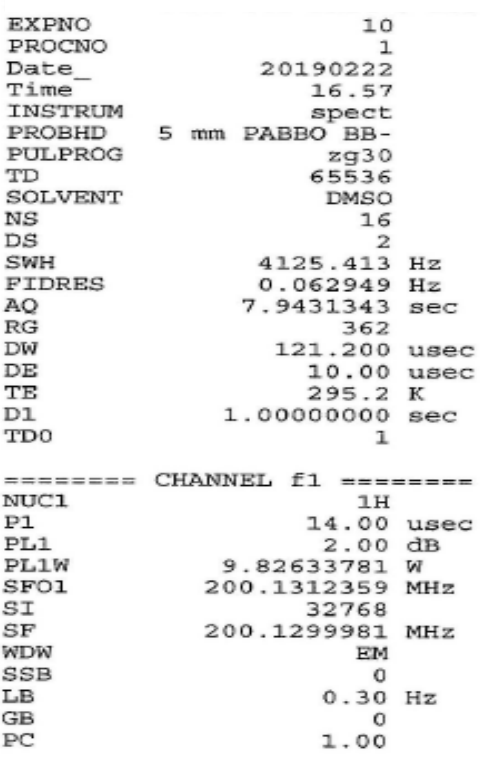




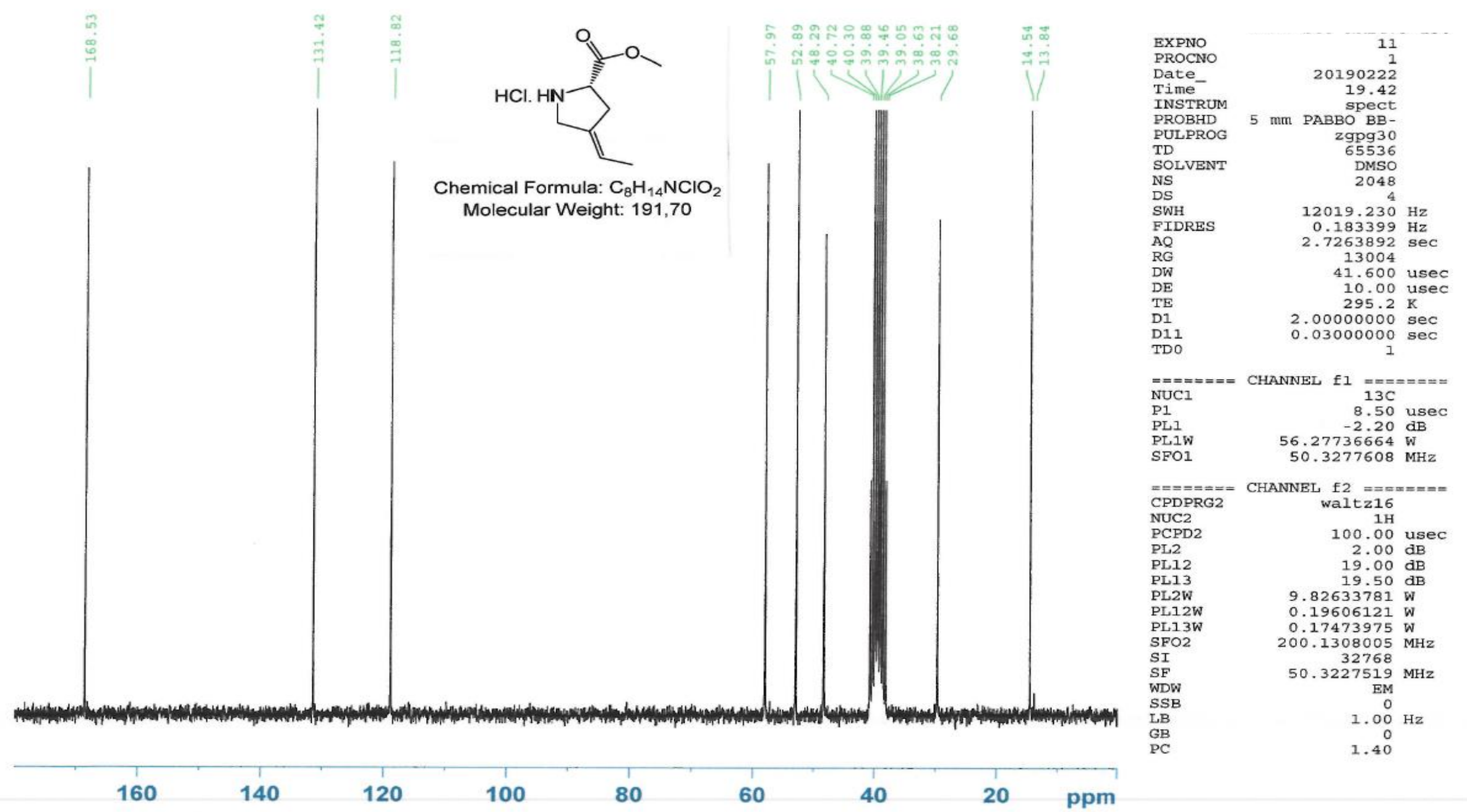




\section{Compound 14: ${ }^{1} \mathrm{H}$ NMR (200 MHz, DMSO d6)}

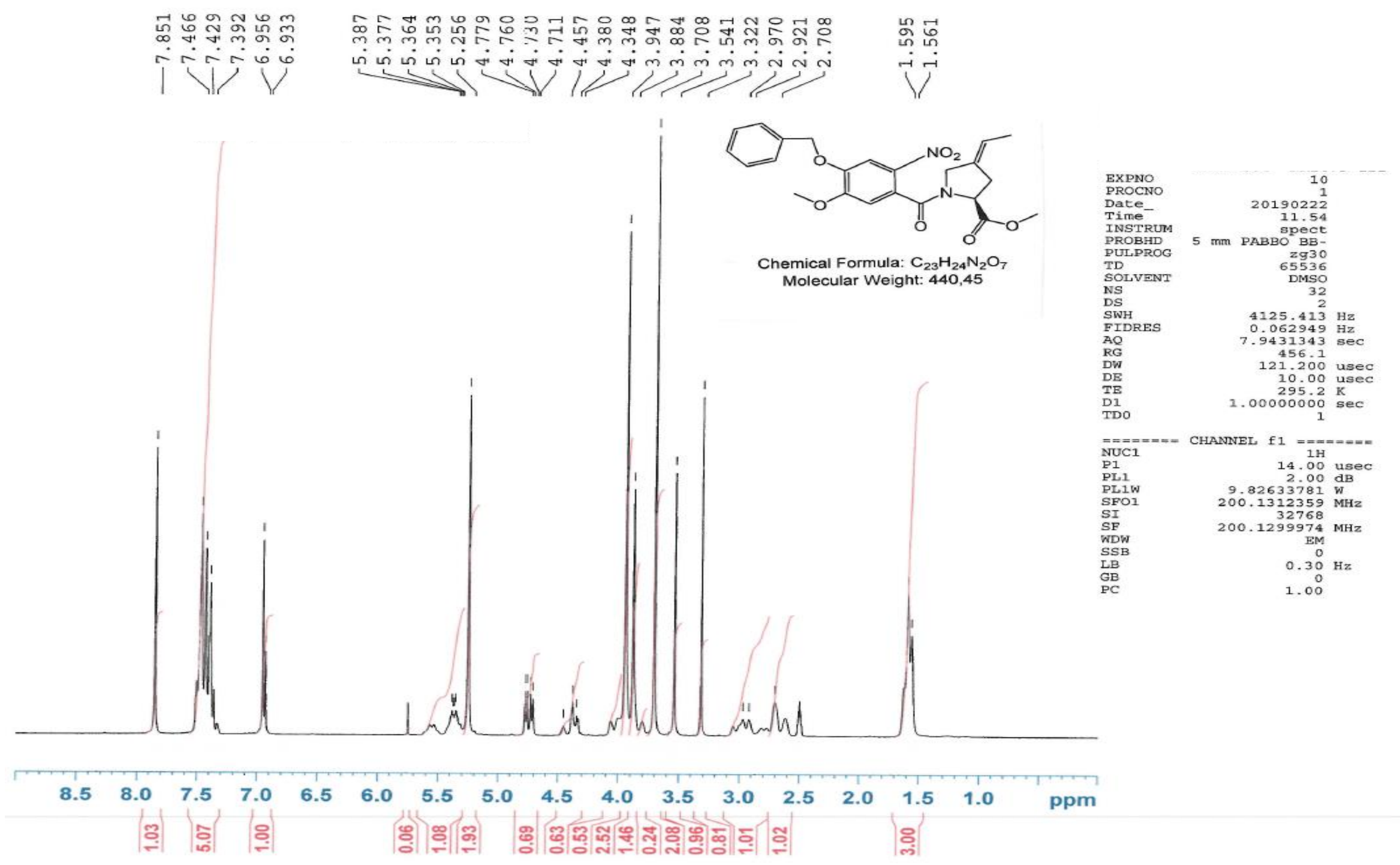




\section{Compound 14: ${ }^{13} \mathrm{C}$ NMR (50 MHz, DMSO d6)}
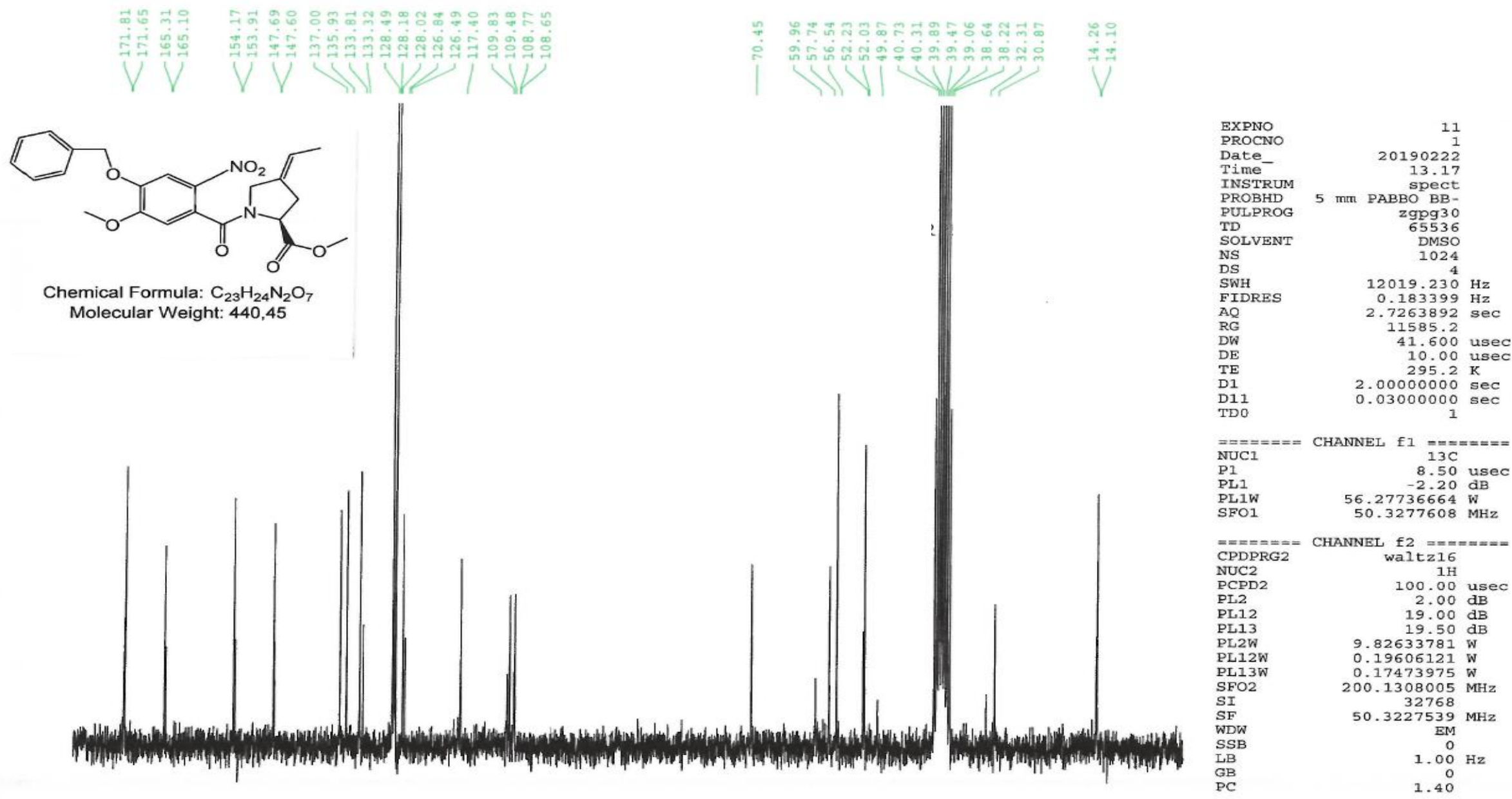

160

140

120

100

80

60

40

20

ppm 
Compound 15: ${ }^{1} \mathrm{H}$ NMR (200 MHz, DMSO d6)

룰

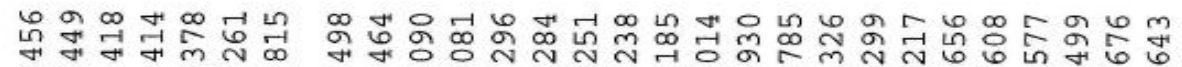

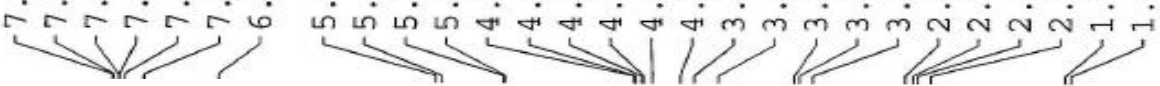

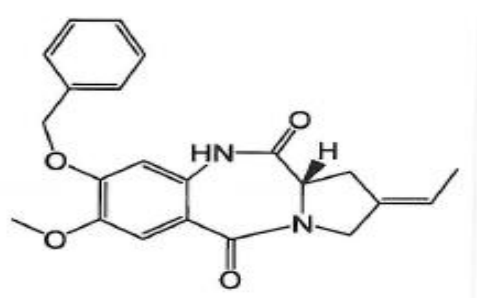

Chemical Formula: $\mathrm{C}_{22} \mathrm{H}_{22} \mathrm{~N}_{2} \mathrm{O}_{4}$ Molecular Weight: 378,43
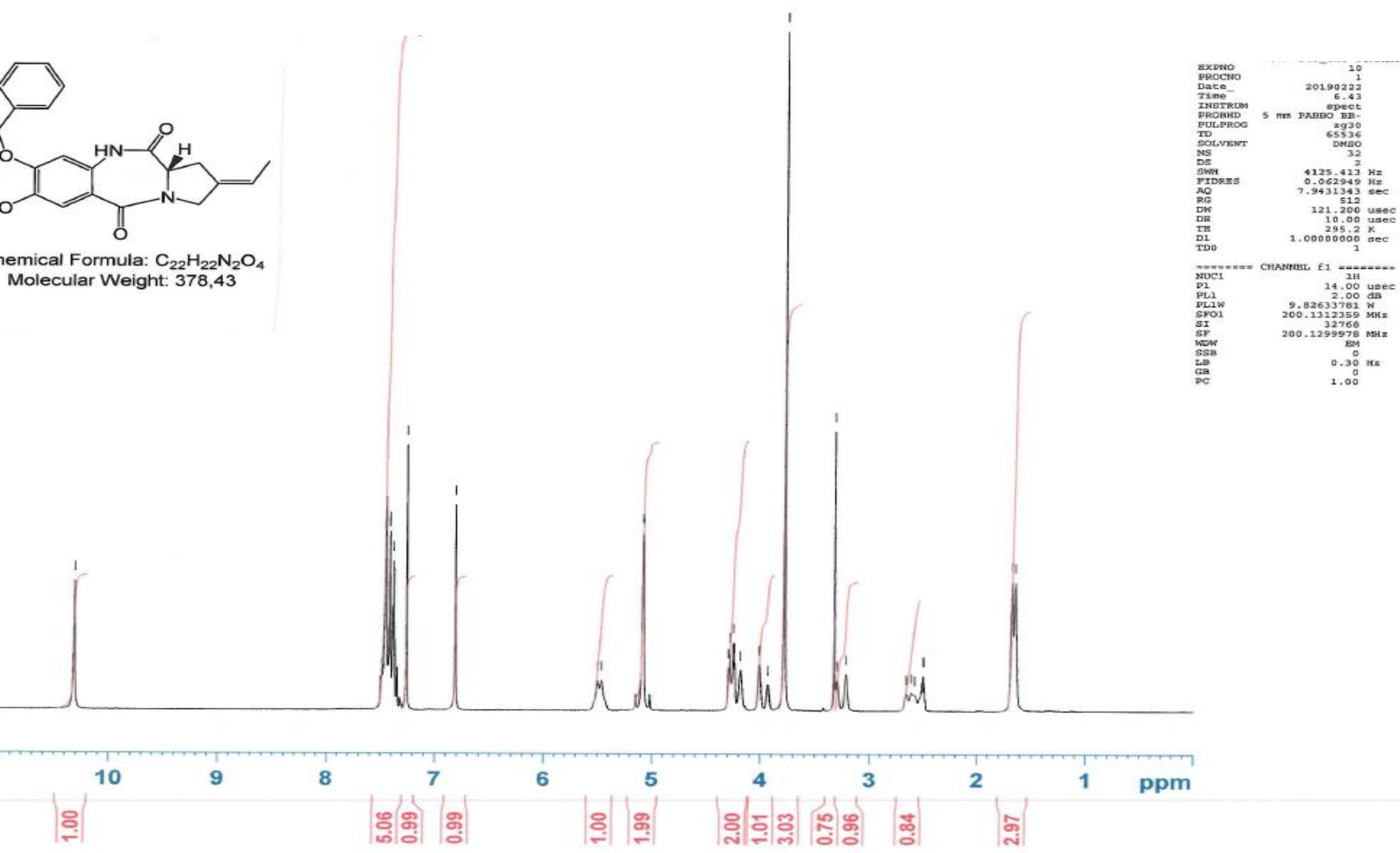
Compound 15: ${ }^{13} \mathrm{C}$ NMR (50 MHz, DMSO d6)
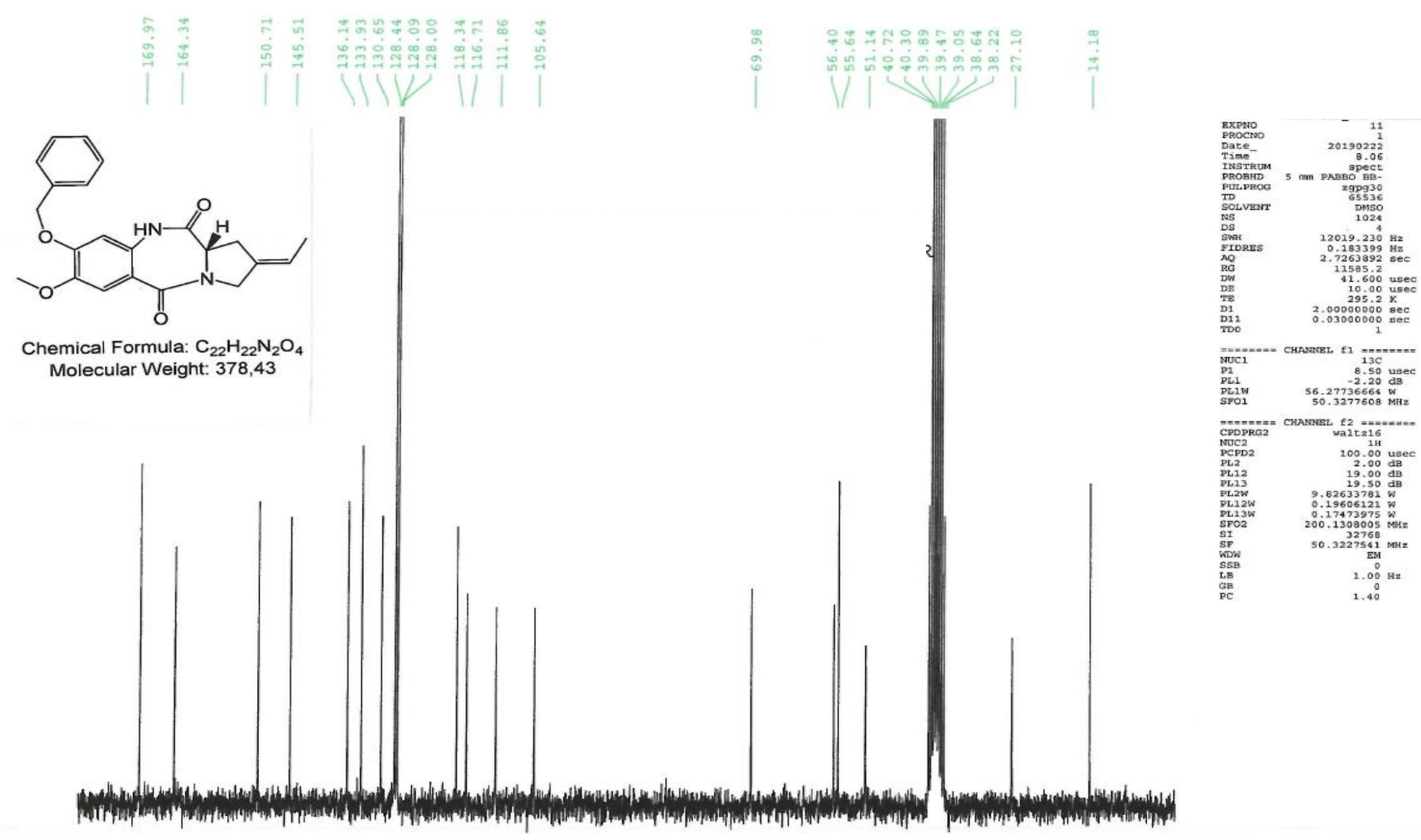


\section{Compound 2 (hemi solvate of THF): ${ }^{1} \mathrm{H}$ NMR (200 MHz, DMSO d6)}

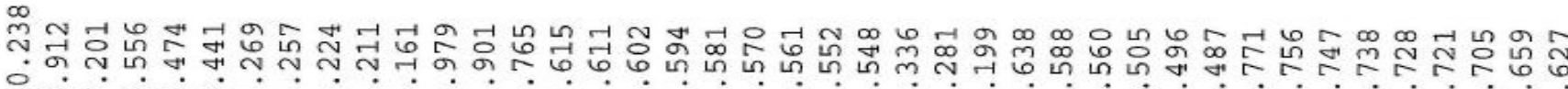

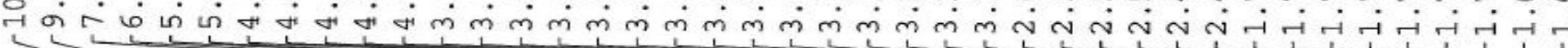

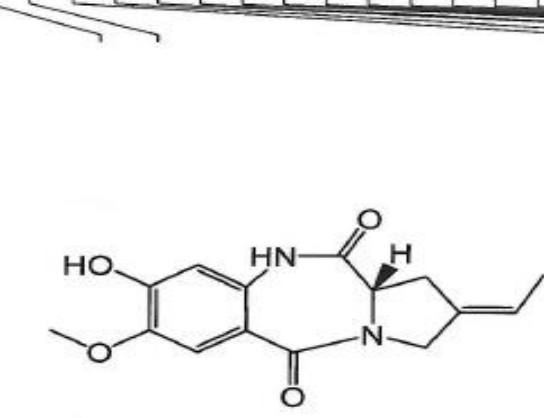

Chemical Formula: $\mathrm{C}_{15} \mathrm{H}_{16} \mathrm{~N}_{2} \mathrm{O}$

Molecular Weight: 288,30
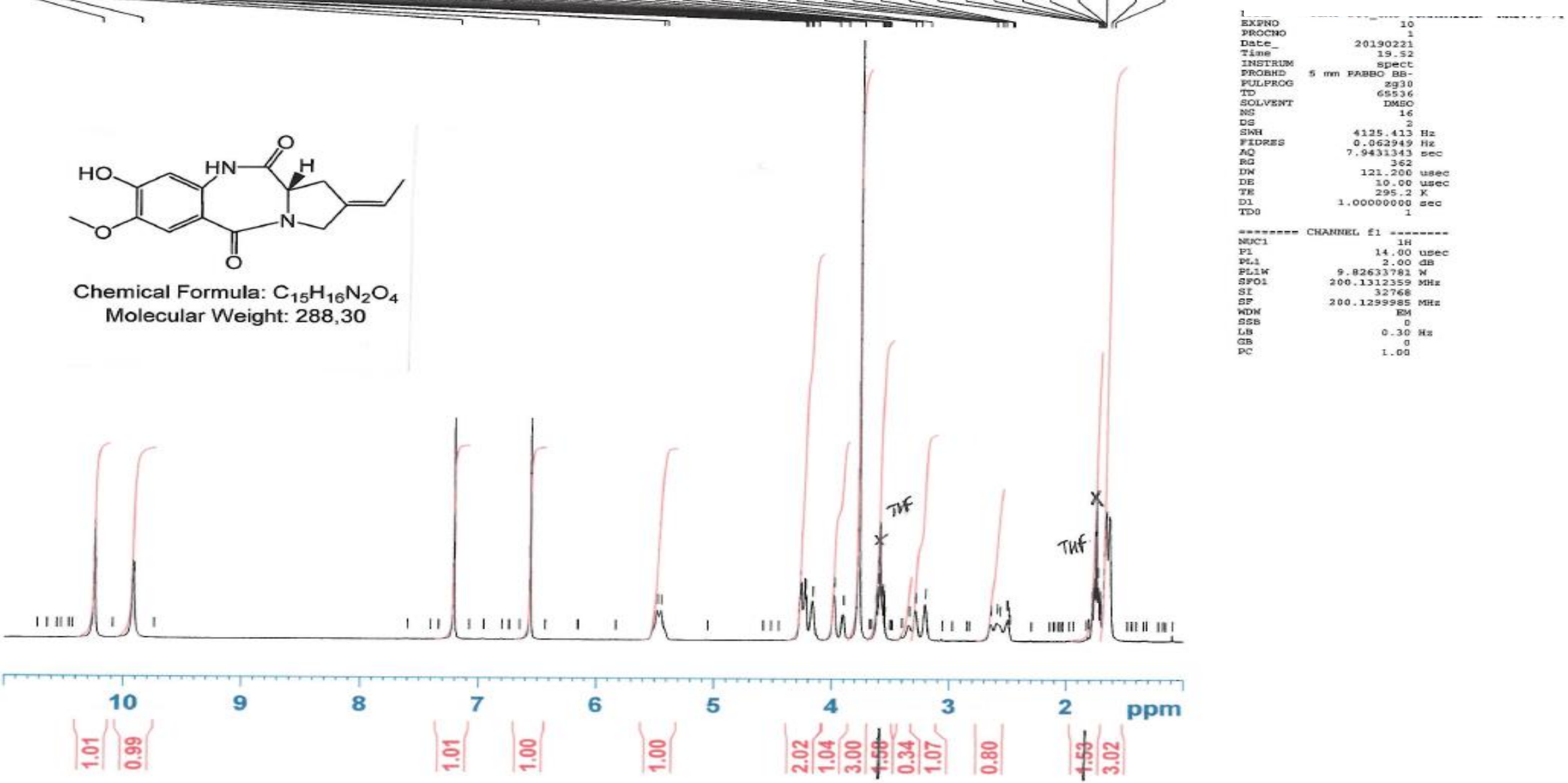


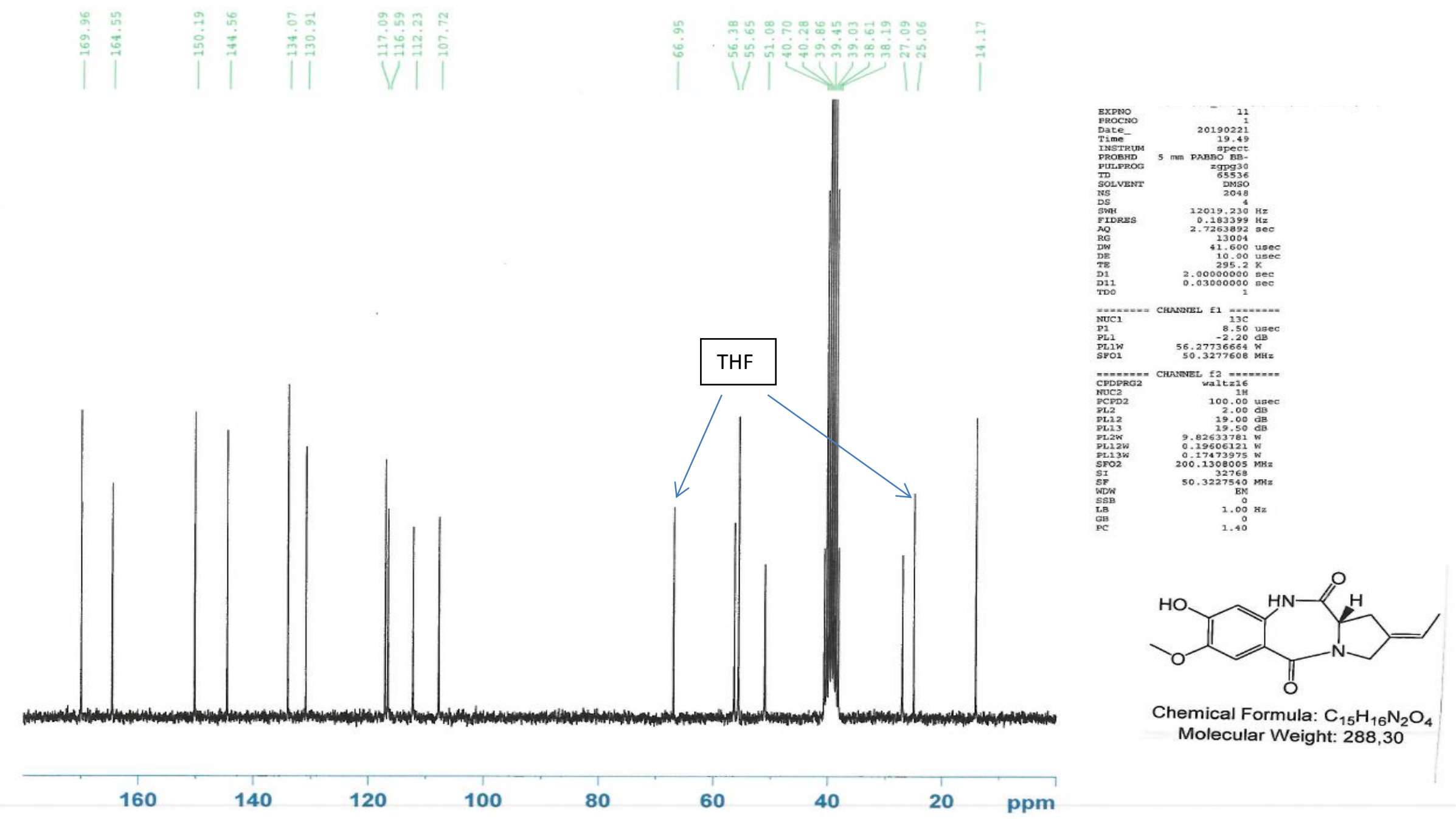


Initial preparative SFC conditions used to separate $E$ and $Z$ isomers of compound 11

\begin{tabular}{|c|l|}
\hline Stationary phase & Chiralpak IC, $20 \mu \mathrm{m}$, packed in a $20 \mathrm{~mm}$ i.d. $\times 250 \mathrm{~mm}$ column \\
\hline Mobile phase & 8 vol.\% of isopropanol (co-solvent) \\
\hline Flow rate & $80 \mathrm{~g} / \mathrm{min}$ \\
\hline Working pressure & $90 \mathrm{bar}$ \\
\hline Feed solution & $50 \mathrm{~g}$ of crude per litre of isopropanol \\
\hline Column loading & $0.106 \% \mathrm{w} / \mathrm{w}$ (ratio crude/CSP) \\
\hline Temperature & $25^{\circ} \mathrm{C}$ \\
\hline UV Detection & $205 \mathrm{~nm}$ \\
\hline Run Time & $4.2 \mathrm{~min}$ \\
\hline
\end{tabular}

$446 \mathrm{~g}$ of crude 11 (E/Z) engaged, $142.7 \mathrm{~g}$ of pure $(E)-11$ obtained i.e. $32 \%$ yield.

Representative chromatogram of run 505

F1 : waste fraction


Preparative HPLC conditions used to separate $E$ and $Z$ isomers of compound 11

\begin{tabular}{|c|l|}
\hline \multicolumn{1}{|c|}{ Stationary phase } & Chiralpak IC $20 \mu \mathrm{m}$ \\
\hline Column dimensions & $250 \times 50 \mathrm{~mm}$ ID. \\
\hline Mobile phase & n-heptane / isopropanol $90 / 10 \mathrm{v} / \mathrm{v}$ \\
\hline Temperature & $35^{\circ} \mathrm{C}$ \\
\hline Flow rate & $7.1 \mathrm{~L} / \mathrm{h}$ \\
\hline Feed concentration & Between $109 \& 124 \mathrm{~g}$ of crude per litre of eluent \\
\hline Acquisition wavelength & $210 \mathrm{~nm}$ \\
\hline Amount injected of crude per run & $1.2 \mathrm{~g}$ (up to $2.4 \mathrm{~g}$ ) \\
\hline Run time (min) & $16 \mathrm{~min}$ (up to $22 \mathrm{~min})$. \\
\hline Pre-concentration bath temp. & $25^{\circ} \mathrm{C}$ \\
\hline Dryness step bath temp. & $30^{\circ} \mathrm{C}$ \\
\hline
\end{tabular}

$880 \mathrm{~g}$ of crude 11 (E/Z) engaged, $275.8 \mathrm{~g}$ of pure (E)-11 obtained i.e. 31\% yield.

\section{Representative chromatogram of run 40 and run 252}

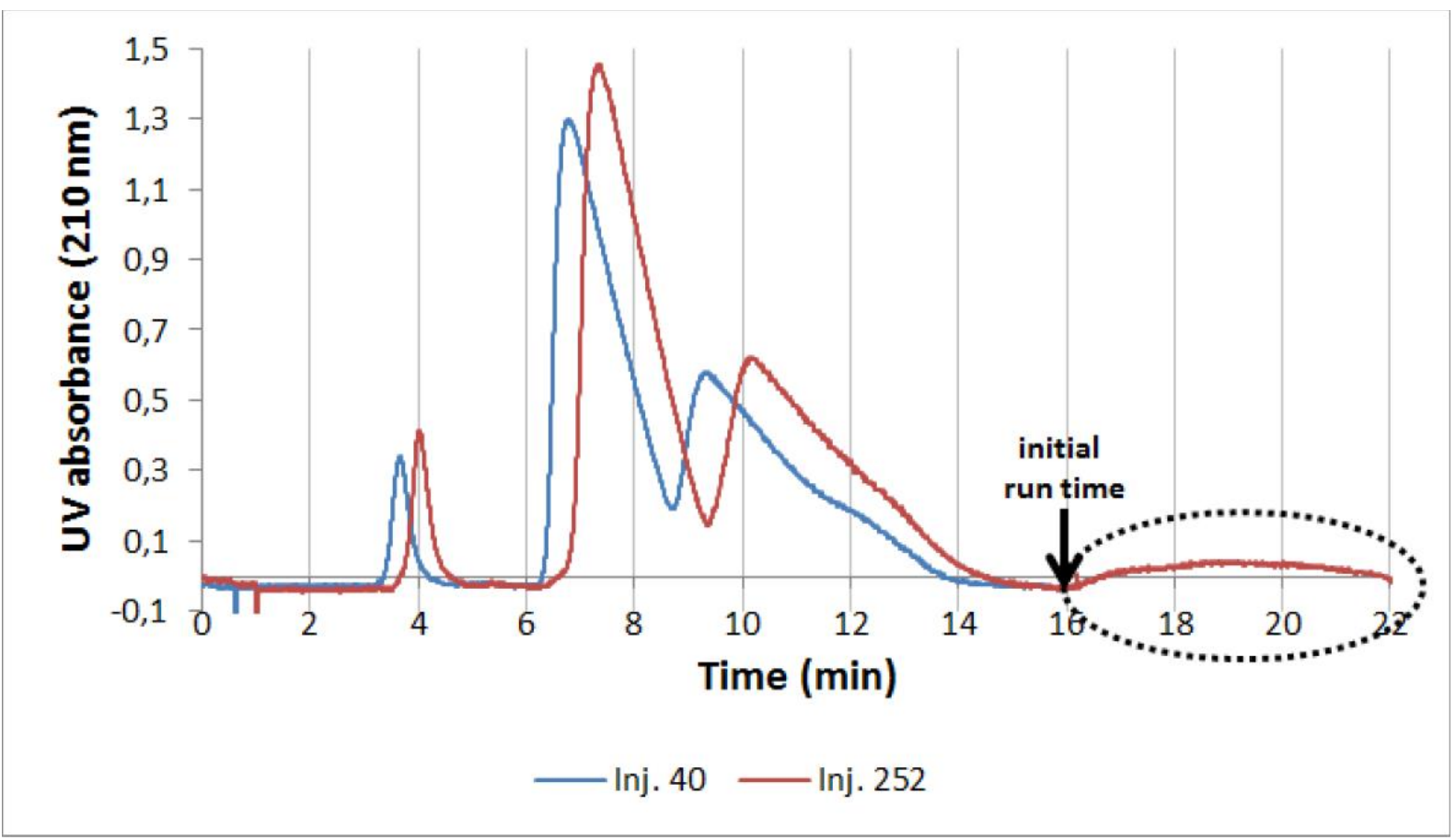


Global yield and Input/output mass balance

\begin{tabular}{|rcc|}
\hline $\begin{array}{r}\text { INPUT - Amount of isomer } \mathrm{E} \\
\text { in the crudes (g) }\end{array}$ & $\mathbf{3 0 3 . 9 \mathrm { g }}$ & $100.0 \%$ \\
\hline $\begin{array}{r}\text { OUTPUT - Amount of isomer } \\
\mathrm{E} \text { in the purified streams }\end{array}$ & $\mathbf{2 7 5 . 8 \mathrm { g }}$ & $\mathbf{9 0 . 8 \%}$ \\
\hline $\begin{array}{r}\text { Amount of isomer } \mathrm{E} \text { in the } \\
\text { waste fractions }\end{array}$ & $23.6 \mathrm{~g}$ & $7.8 \%$ \\
\hline $\begin{array}{r}\text { Amount of isomer E in the } \\
\text { distillates }\end{array}$ & $0.3 \mathrm{~g}$ & $0.1 \%$ \\
\hline Unknown losses & $4.2 \mathrm{~g}$ & $1.3 \%$ \\
\hline
\end{tabular}

Process comparability and industrial scale-up considerations

\begin{tabular}{|c|c|c|}
\hline & SFC & HPLC \\
\hline Productivity (KKD) & 0.36 & 0.55 \\
\hline $\begin{array}{c}\text { (E)-11 recovery rate } \\
\text { Mobile phase recycling } \\
\text { rate (expected) }\end{array}$ & $32 \%$ & $31 \%$ \\
\hline $\begin{array}{c}\text { Column back pressure } \\
\text { (expected ranges) }\end{array}$ & $82-92 \%$ & $80-90 \%$ \\
\hline $\begin{array}{c}\text { Equipment scalability } \\
\text { OK for column with internal } \\
\text { diameter } \leq 80 \mathrm{~mm}\end{array}$ & No size restriction \\
\hline
\end{tabular}




\section{X-ray crystallography data for (+)-oxo-tomaymycin}

Crystals of (+)-oxo-tomaymycin were obtained by slow evaporation method, from a methanol solution. The X-ray data was collected to establish the relative stereochemistry (see Figure S1).

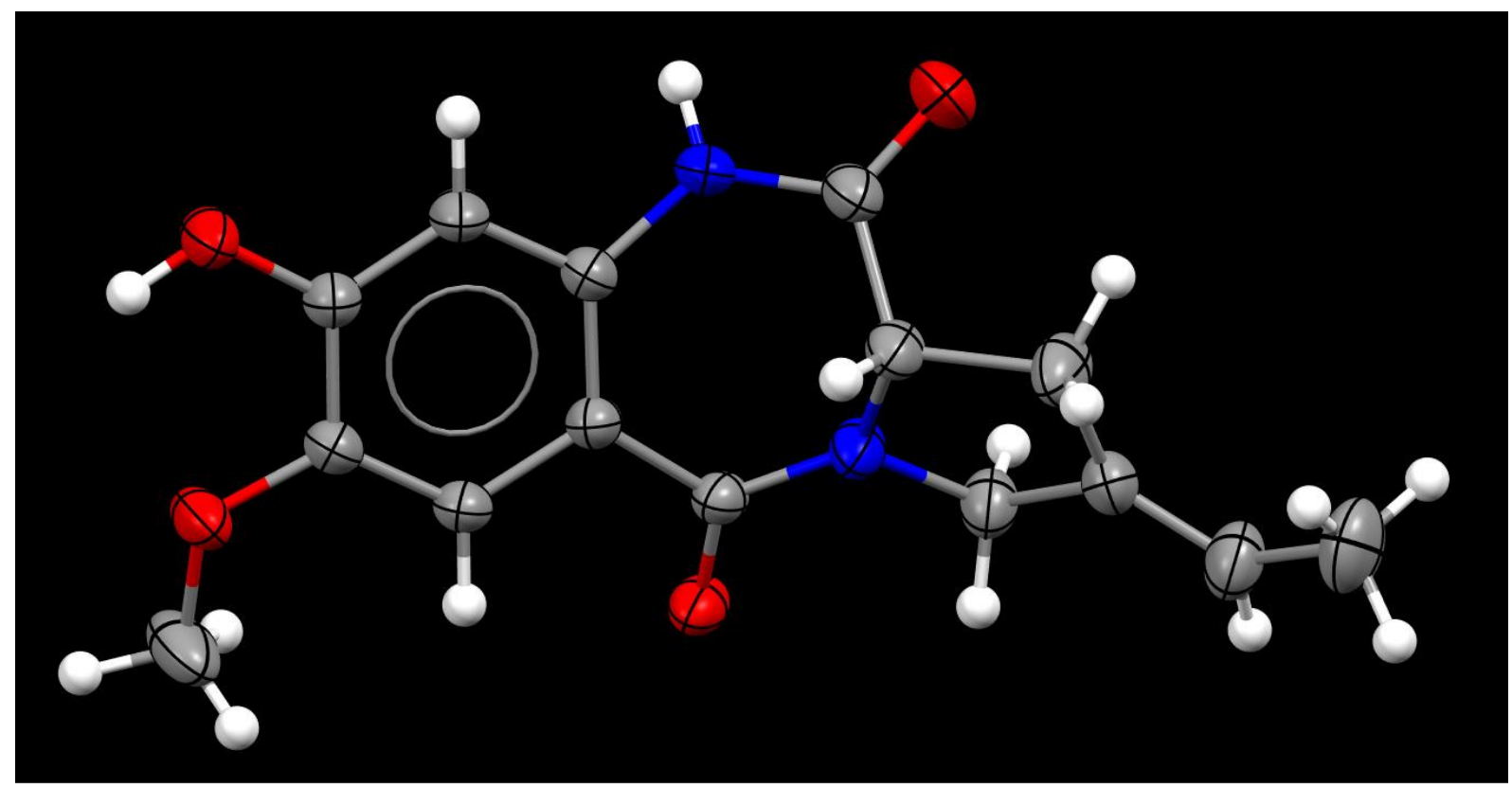

Figure S1: ORTEP diagram representation showing molecular configuration of (+)-oxo-tomaymycin 2. (CCDC Deposition Number 1972508); carbon in grey, hydrogen in white, oxygen in red, nitrogen in blue.

A suitable crystal of $\mathrm{C}_{15} \mathrm{H}_{16} \mathrm{~N}_{2} \mathrm{O}_{4}$ was selected and measured on a Bruker AXS D8 Venture diffractometer equipped with a Mo Incoatec micro-focus source I $\mu$ S 3.0 and a Photon II CPAD detector. The crystal was kept at 302 (1) K during data collection. Using Bruker Apex3 software suite 2018 the structure was solved with the XT [Acta Cryst. (2015). A71, 3-8. (2015)] structure solution program using Intrinsic Phasing and refined with the XL [Acta Cryst. A64, 112-122. (2008) \& Acta Cryst. (2015). C71, 3-8] refinement package using Least Squares minimization.

The following report was created with Olex2 software (Dolomanov, O.V., Bourhis, L.J., Gildea, R.J, Howard, J.A.K. \& Puschmann, H. (2009), J. Appl. Cryst. 42, 339-341).

Table S1: Crystal data and structure refinement for (+)-oxo-Tomaymycin

\begin{tabular}{|l|l|}
\hline Empirical formula & $\mathrm{C}_{15} \mathrm{H}_{16} \mathrm{~N}_{2} \mathrm{O}_{4}$ \\
\hline Formula weight & 288.30 \\
\hline Temperature/K & $302(2)$ \\
\hline Crystal system & monoclinic \\
\hline Space group & $\mathrm{P} 21$ \\
\hline $\mathrm{a} / \AA$ & $6.9821(2)$ \\
\hline $\mathrm{b} / \AA$ & $8.6527(3)$ \\
\hline c/ $\AA$ & $11.9259(3)$ \\
\hline$\alpha /{ }^{\circ}$ & 90 \\
\hline$\beta /{ }^{\circ}$ & $105.7610(10)$ \\
\hline$\gamma /{ }^{\circ}$ & 90 \\
\hline Volume $/ \AA^{3}$ & $693.40(4)$ \\
\hline
\end{tabular}




\begin{tabular}{|c|c|}
\hline $\mathrm{Z}$ & 2 \\
\hline$\rho_{\text {calc } g / \mathrm{cm}^{3}}$ & 1.381 \\
\hline$\mu / \mathrm{mm}^{-1}$ & 0.101 \\
\hline $\mathrm{F}(000)$ & 304.0 \\
\hline Crystal size $/ \mathrm{mm}^{3}$ & $0.04 \times 0.15 \times 0.15$ \\
\hline Radiation & $\operatorname{MoK} \alpha(\lambda=0.71073)$ \\
\hline $2 \Theta$ range for data collection $/^{\circ}$ & 5.896 to 61.024 \\
\hline Index ranges & $-9 \leq \mathrm{h} \leq 9,-12 \leq \mathrm{k} \leq 12,-17 \leq 1 \leq 17$ \\
\hline Reflections collected & 27529 \\
\hline Independent reflections & $4184\left[\mathrm{R}_{\mathrm{int}}=0.0277, \mathrm{R}_{\mathrm{sigma}}=0.0250\right]$ \\
\hline Data/restraints/parameters & $4184 / 1 / 200$ \\
\hline Goodness-of-fit on $\mathrm{F}^{2}$ & 1.061 \\
\hline Final $R$ indexes $[\mathrm{I}>=2 \sigma(\mathrm{I})]$ & $\mathrm{R}_{1}=0.0349, \mathrm{wR}_{2}=0.1016$ \\
\hline Final $\mathrm{R}$ indexes [all data] & $\mathrm{R}_{1}=0.0372, \mathrm{wR}_{2}=0.1044$ \\
\hline Largest diff. peak/hole / e $\AA^{-3}$ & $0.25 /-0.15$ \\
\hline Flack parameter $*$ & $-0.01(19)$ \\
\hline
\end{tabular}

*: from 1747 selected quotients (Parsons' method see Parsons, S., Flack, H. D., \& Wagner, T. (2013). Use of intensity quotients and differences in absolute structure refinement. Acta crystallographica Section B, Structural science, crystal engineering and materials, 69(Pt 3), 249-259.)

Table S2: Fractional Atomic Coordinates $\left(\times 10^{4}\right)$ and Equivalent Isotropic Displacement Parameters $\left(\AA^{2} \times 10^{3}\right)$. Ueq is defined as $1 / 3$ of the trace of the orthogonalised Uı tensor.

\begin{tabular}{|l|r|r|r|r|}
\hline Atom & \multicolumn{1}{|c|}{$\boldsymbol{x}$} & \multicolumn{1}{|c|}{$\boldsymbol{y}$} & \multicolumn{1}{c|}{$\boldsymbol{z}$} & \multicolumn{1}{c|}{$\mathbf{U}(\mathbf{e q})$} \\
\hline O001 & $8725.3(16)$ & $6688.1(16)$ & $2470.0(9)$ & $37.4(3)$ \\
\hline O2 & $4953(2)$ & $3197.4(17)$ & $-733.7(12)$ & $43.3(3)$ \\
\hline O003 & $1421.9(18)$ & $2701.3(18)$ & $-281.7(11)$ & $44.3(3)$ \\
\hline N004 & $3197(2)$ & $5683.1(19)$ & $3167.3(12)$ & $37.2(3)$ \\
\hline O005 & $3468(2)$ & $6560(3)$ & $4981.0(13)$ & $60.7(4)$ \\
\hline N6 & $7485.3(19)$ & $6270.9(18)$ & $4003.4(11)$ & $35.5(3)$ \\
\hline C007 & $4735(2)$ & $3762.8(19)$ & $291.5(13)$ & $32.3(3)$ \\
\hline C008 & $3883(2)$ & $4960.8(19)$ & $2280.8(12)$ & $31.3(3)$ \\
\hline C009 & $5796(2)$ & $5150.3(18)$ & $2130.0(12)$ & $29.6(3)$ \\
\hline C00A & $2852(2)$ & $3507.3(19)$ & $478.9(14)$ & $33.7(3)$ \\
\hline C00B & $4217(2)$ & $5909(2)$ & $4295.0(14)$ & $37.0(3)$ \\
\hline C00C & $6168(2)$ & $4540.4(19)$ & $1117.4(13)$ & $32.1(3)$ \\
\hline C00D & $7429(2)$ & $6091.1(18)$ & $2878.0(12)$ & $30.2(3)$ \\
\hline C00E & $2456(2)$ & $4120(2)$ & $1462.5(14)$ & $36.2(3)$ \\
\hline C00F & $9157(2)$ & $6552(2)$ & $5966.6(13)$ & $36.6(3)$ \\
\hline C00G & $6346(2)$ & $5319(2)$ & $4616.5(13)$ & $35.3(3)$ \\
\hline C00H & $7413(3)$ & $5498(3)$ & $5911.9(14)$ & $48.6(5)$ \\
\hline C00I & $10747(3)$ & $6714(3)$ & $6865.5(16)$ & $47.2(4)$ \\
\hline C00J & $8893(3)$ & $7305(2)$ & $4797.1(14)$ & $38.7(3)$ \\
\hline C00K & $6545(4)$ & $3769(3)$ & $-1130(2)$ & $61.4(6)$ \\
\hline C00L & $11058(4)$ & $5912(3)$ & $8008.9(19)$ & $61.6(6)$ \\
\hline
\end{tabular}


Table S3: Anisotropic Displacement Parameters $\left(\AA^{2} \times 10^{3}\right)$. The Anisotropic displacement factor exponent takes the form: $-2 \pi^{2}\left[h^{2} a * 2 U_{11}+2 h k a * b * U_{12}+\ldots\right]$.

\begin{tabular}{|l|r|r|r|r|r|r|}
\hline Atom & \multicolumn{1}{|c|}{$\mathbf{U}_{\mathbf{1 1}}$} & \multicolumn{1}{c|}{$\mathbf{U}_{\mathbf{2 2}}$} & \multicolumn{1}{|c|}{$\mathbf{U}_{\mathbf{3 3}}$} & \multicolumn{1}{|c|}{$\mathbf{U}_{\mathbf{2 3}}$} & \multicolumn{1}{c|}{$\mathbf{U}_{\mathbf{1 3}}$} & \multicolumn{1}{c|}{$\mathbf{U}_{\mathbf{1 2}}$} \\
\hline O001 & $28.8(5)$ & $55.1(7)$ & $28.7(5)$ & $4.5(5)$ & $8.8(4)$ & $-8.2(5)$ \\
\hline O2 & $38.9(6)$ & $57.6(7)$ & $38.3(6)$ & $-16.0(6)$ & $18.8(5)$ & $-9.9(5)$ \\
\hline O003 & $33.0(6)$ & $65.0(8)$ & $36.9(6)$ & $-16.6(6)$ & $12.8(5)$ & $-11.5(5)$ \\
\hline N004 & $25.1(6)$ & $54.6(8)$ & $33.8(6)$ & $-7.4(6)$ & $11.1(5)$ & $0.9(5)$ \\
\hline O005 & $48.1(7)$ & $93.7(12)$ & $43.9(7)$ & $-23.3(8)$ & $18.4(6)$ & $5.9(8)$ \\
\hline N6 & $30.6(6)$ & $50.4(8)$ & $26.1(5)$ & $0.3(5)$ & $8.6(4)$ & $-9.0(5)$ \\
\hline C007 & $29.0(6)$ & $39.7(7)$ & $30.3(7)$ & $-2.3(6)$ & $11.5(5)$ & $0.4(5)$ \\
\hline C008 & $27.4(6)$ & $40.0(7)$ & $28.1(6)$ & $-1.8(5)$ & $10.1(5)$ & $0.7(5)$ \\
\hline C009 & $24.6(6)$ & $37.2(7)$ & $27.2(6)$ & $0.1(5)$ & $7.5(5)$ & $0.1(5)$ \\
\hline C00A & $28.5(6)$ & $41.9(8)$ & $31.3(7)$ & $-3.8(6)$ & $9.2(5)$ & $-2.5(6)$ \\
\hline C00B & $33.2(7)$ & $47.5(8)$ & $33.5(7)$ & $-5.7(6)$ & $14.6(6)$ & $-4.5(6)$ \\
\hline C00C & $25.5(6)$ & $41.5(7)$ & $30.9(6)$ & $-1.2(6)$ & $10.5(5)$ & $-0.3(5)$ \\
\hline C00D & $24.6(6)$ & $40.2(7)$ & $26.2(6)$ & $3.2(5)$ & $7.8(5)$ & $0.2(5)$ \\
\hline C00E & $25.5(6)$ & $51.9(9)$ & $33.2(7)$ & $-5.8(6)$ & $11.4(5)$ & $-5.4(6)$ \\
\hline C00F & $37.0(7)$ & $44.7(8)$ & $28.2(6)$ & $-2.9(6)$ & $9.1(5)$ & $0.7(6)$ \\
\hline C00G & $31.7(7)$ & $47.9(8)$ & $28.0(6)$ & $2.6(6)$ & $11.3(5)$ & $-5.7(6)$ \\
\hline C00H & $42.6(9)$ & $75.8(13)$ & $26.9(7)$ & $6.3(8)$ & $8.8(6)$ & $-11.6(9)$ \\
\hline C00I & $45.9(9)$ & $56.3(11)$ & $34.1(8)$ & $-2.9(8)$ & $2.1(7)$ & $-8.1(8)$ \\
\hline C00J & $39.2(8)$ & $46.4(8)$ & $29.7(7)$ & $-2.3(6)$ & $7.8(6)$ & $-9.2(6)$ \\
\hline C00K & $59.7(12)$ & $83.5(16)$ & $53.0(11)$ & $-22.9(11)$ & $35.8(10)$ & $-23.8(12)$ \\
\hline C00L & $67.8(14)$ & $70.1(14)$ & $36.4(9)$ & $4.7(9)$ & $-3.5(9)$ & $-1.6(11)$ \\
\hline
\end{tabular}

Table S4: Bond Lengths.

\begin{tabular}{|c|c|c|c|c|c|}
\hline Atom & Atom & Length/ & Atom & Atom & Length/Å \\
\hline O001 & $\mathrm{COOD}$ & $1.2497(17)$ & $\mathrm{C} 008$ & $\mathrm{COOE}$ & $1.395(2)$ \\
\hline $\mathrm{O} 2$ & $\mathrm{C} 007$ & $1.3632(18)$ & $\mathrm{C} 008$ & $\mathrm{C009}$ & $1.4047(19)$ \\
\hline $\mathrm{O} 2$ & $\mathrm{COOK}$ & $1.411(2)$ & C009 & $\mathrm{COOC}$ & $1.4051(19)$ \\
\hline $\mathrm{O} 003$ & $\mathrm{COOA}$ & $1.3471(19)$ & $\mathrm{C} 009$ & $\mathrm{COOD}$ & $1.485(2)$ \\
\hline N004 & $\mathrm{COOB}$ & $1.354(2)$ & $\mathrm{C} 00 \mathrm{~A}$ & $\mathrm{COOE}$ & $1.382(2)$ \\
\hline N004 & $\mathrm{C008}$ & $1.4189(19)$ & $\mathrm{CO0B}$ & C00G & $1.519(2)$ \\
\hline O005 & COOB & $1.222(2)$ & $\mathrm{COOF}$ & $\mathrm{COOI}$ & $1.325(2)$ \\
\hline N6 & C00D & $1.3412(18)$ & $\mathrm{C} 00 \mathrm{~F}$ & $\mathrm{COOJ}$ & $1.505(2)$ \\
\hline N6 & $\mathrm{COOJ}$ & $1.469(2)$ & $\mathrm{COOF}$ & $\mathrm{COOH}$ & $1.508(3)$ \\
\hline N6 & C00G & $1.4703(19)$ & $\mathrm{CO0G}$ & $\mathrm{COOH}$ & $1.530(2)$ \\
\hline $\mathrm{C} 007$ & $\mathrm{COOC}$ & $1.374(2)$ & $\mathrm{COOI}$ & $\mathrm{COOL}$ & $1.493(3)$ \\
\hline $\mathrm{C} 007$ & $\mathrm{COOA}$ & $1.409(2)$ & & & \\
\hline
\end{tabular}


Table S5: Bond Angles.

\begin{tabular}{|l|l|l|l|l|l|l|l|r|}
\hline Atom & Atom & Atom & Angle $^{\circ}$ & & Atom & Atom & Atom & Angle $^{\circ}$ \\
\hline C007 & O2 & C00K & $117.71(14)$ & & O005 & C00B & N004 & $121.73(16)$ \\
\hline C00B & N004 & C008 & $127.61(14)$ & & O005 & C00B & C00G & $123.61(16)$ \\
\hline C00D & N6 & C00J & $123.24(13)$ & & N004 & C00B & C00G & $114.65(13)$ \\
\hline C00D & N6 & C00G & $124.05(13)$ & & C007 & C00C & C009 & $122.11(13)$ \\
\hline C00J & N6 & C00G & $112.25(12)$ & & O001 & C00D & N6 & $120.95(14)$ \\
\hline O2 & C007 & C00C & $125.58(13)$ & & O001 & C00D & C009 & $120.78(13)$ \\
\hline O2 & C007 & C00A & $114.96(13)$ & & N6 & C00D & C009 & $118.27(12)$ \\
\hline C00C & C007 & C00A & $119.46(14)$ & & C00A & C00E & C008 & $121.60(14)$ \\
\hline C00E & C008 & C009 & $119.64(13)$ & & C00I & C00F & C00J & $124.67(17)$ \\
\hline C00E & C008 & N004 & $115.42(13)$ & & C00I & C00F & C00H & $126.05(17)$ \\
\hline C009 & C008 & N004 & $124.71(14)$ & & C00J & C00F & C00H & $109.11(13)$ \\
\hline C008 & C009 & C00C & $118.04(13)$ & & N6 & C00G & C00B & $108.10(13)$ \\
\hline C008 & C009 & C00D & $126.06(13)$ & & N6 & C00G & C00H & $105.33(13)$ \\
\hline C00C & C009 & C00D & $115.54(12)$ & & C00B & C00G & C00H & $113.31(14)$ \\
\hline O003 & C00A & C00E & $119.16(13)$ & & C00F & C00H & C00G & $105.72(13)$ \\
\hline O003 & C00A & C007 & $121.83(14)$ & & C00F & C00I & C00L & $124.9(2)$ \\
\hline C00E & C00A & C007 & $119.01(14)$ & & N6 & C00J & C00F & $103.02(13)$ \\
\hline
\end{tabular}

Table S6: Hydrogen Atom Coordinates $\left(\AA \times 10^{4}\right)$ and Isotropic Displacement Parameters $\left(\AA^{2} \times 10^{3}\right)$.

\begin{tabular}{|l|r|r|r|r|}
\hline Atom & \multicolumn{1}{|c|}{$\boldsymbol{x}$} & \multicolumn{1}{c|}{$\boldsymbol{y}$} & \multicolumn{1}{c|}{$\boldsymbol{z}$} & \multicolumn{1}{c|}{$\mathbf{U}(\mathbf{e q})$} \\
\hline H3 & $1700(60)$ & $2400(50)$ & $-930(30)$ & $92(11)$ \\
\hline H4 & $2000(40)$ & $5960(30)$ & $2990(20)$ & $53(7)$ \\
\hline H00C & 7423.32 & 4667.16 & 1002.46 & 38 \\
\hline H00E & 1207.32 & 3967.96 & 1581.75 & 43 \\
\hline H00G & 6370.69 & 4234.58 & 4383.59 & 42 \\
\hline H00A & 6530.66 & 5949.24 & 6325.59 & 58 \\
\hline H00B & 7866.94 & 4502.32 & 6256.89 & 58 \\
\hline H00I & 11743.91 & 7378.19 & 6777.76 & 57 \\
\hline H00D & 8346.94 & 8338.06 & 4785.93 & 46 \\
\hline H00F & 10145.39 & 7362.37 & 4594.36 & 46 \\
\hline H00H & 6341.68 & 3498.13 & -1934.79 & 92 \\
\hline H00J & 7770.73 & 3324.99 & -676.11 & 92 \\
\hline H00K & 6606.36 & 4873.3 & -1049.95 & 92 \\
\hline H00L & 10290.64 & 4975.82 & 7901.54 & 92 \\
\hline H00M & 10639.45 & 6574.7 & 8543.17 & 92 \\
\hline H00N & 12442.69 & 5668.05 & 8317.19 & 92 \\
\hline
\end{tabular}

